\title{
Specific microRNAs are involved in the reno-protective effects of sevoflurane preconditioning and ischemic preconditioning against ischemia reperfusion injury in rats
}

\author{
MAKIKO YAMAMOTO, TOMONORI MORITA, MASASHI ISHIKAWA and ATSUHIRO SAKAMOTO \\ Department of Anesthesiology and Pain Medicine, Graduate School of Medicine, \\ Nippon Medical School, Tokyo 1138602, Japan
}

Received October 22, 2019; Accepted January 8, 2020

DOI: $10.3892 / \mathrm{ijmm} .2020 .4477$

\begin{abstract}
The kidneys are prone to developing ischemia reperfusion injury (IRI) following certain renal surgeries and cardiovascular surgeries requiring cardiac arrest. Sevoflurane and ischemic preconditioning reportedly alleviate IRI, which is mediated via microRNAs. The present study compared anesthetic preconditioning (APC) and ischemic preconditioning (IPC) on microRNAs, which promote cell-survival pathways in rats in a randomized controlled study. After undergoing right nephrectomy under general anesthesia, male Wistar rats $(336 \pm 24 \mathrm{~g})$ and were divided into four groups (IRI, APC, IPC and sham; $n=7$ each). The IRI group underwent $45 \mathrm{~min}$ clamping of the left renal vasculature, followed by $4 \mathrm{~h}$ of reperfusion. APC involved exposure to one minimum alveolar concentration sevoflurane for $15 \mathrm{~min}$. IPC included three cycles of two-min clamping and five-min reperfusion. Blood and renal biopsy samples were assessed postoperatively to measure serum creatinine and to analyze renal microRNA (miR) expression using reverse transcription-quantitative polymerase chain reaction (RT-qPCR) testing and their target pathways with Ingenuity Pathway Analysis ${ }^{\mathrm{TM}}$. The present study found that serum creatinine values in APC $(0.71 \pm 0.08 \mathrm{mg} / \mathrm{dl})$ and IPC $(0.73 \pm 0.1 \mathrm{mg} / \mathrm{dl})$ groups were lower than in the IRI group $(0.96 \pm 0.13 \mathrm{mg} / \mathrm{dl} ; \mathrm{P}<0.05)$, indicating amelioration of IRI by APC and IPC. RT-qPCR followed by pathway analysis
\end{abstract}

Correspondence to: Dr Makiko Yamamoto, Department of Anesthesiology and Pain Medicine, Graduate School of Medicine, Nippon Medical School, 1-1-5 Sendagi, Bunkyo-ku, Tokyo 1138602, Japan

E-mail: m-yamamoto@nms.ac.jp

Abbreviations: Akt, protein kinase B; APC, anesthetic preconditioning; IPA, Ingenuity Pathway Analysis; IPC, ischemic preconditioning; IRI, ischemia reperfusion injury; PI3K, phosphoinositide 3-kinase; PTEN, phosphatase and tensin homologue deleted from chromosome 10

Key words: phosphorylated protein kinase B, ischemia reperfusion injury, ischemic preconditioning, kidney, microRNA, sevoflurane indicated that APC and IPC affect 'protein kinase B (Akt)'. APC promoted miR-17-3p and suppressed miR-27a. IPC promoted miR-19a. All the miRs were predicted to regulate phosphorylated Akt, which promotes cell-protection. Western blot analysis showed that expression of phosphorylated Akt increased and phosphatase and tensin homologue deleted from chromosome 10 (PTEN) decreased following APC and IPC. The present study concluded that APC and IPC affect different miRs, although they are estimated to similarly promote the PTEN/phosphoinositide 3-kinase/Akt signaling pathway, resulting in reno-protection.

\section{Introduction}

In certain renal surgeries, including partial nephrectomy and renal transplantation, the renal vasculature is temporarily clamped followed by vascular re-perfusion to preserve the kidney. The procedure might cause renal ischemia reperfusion injury (IRI), which is one of the major causes of postoperative acute renal dysfunction (1). Postoperative renal prognosis is a particularly important factor following such surgeries $(2,3)$. IRI can also occur following cardiovascular surgeries that require cardiac arrest during cardiopulmonary bypass, because of the associated non-physiological circulation $(4,5)$.

IRI reportedly consists of numerous stages, including transcriptional reprogramming during ischemia, followed by an autoimmune inflammatory reaction, vascular leakage and promotion of cell death, including apoptosis during reperfusion (6). Alleviation of IRI improves the prognosis of the preserved kidney (7). Previous animal studies reported that ischemic preconditioning (IPC) alleviates renal IRI $(8,9)$ and numerous clinical studies have elucidated the IPC effects on the kidney. Furthermore, these surgeries are performed under general anesthesia. Administration of certain anesthetics, which is considered anesthetic preconditioning (APC), also reportedly alleviates renal IRI in rats (10-12). Specifically, sevoflurane APC was reported to be effective in alleviating renal IRI both clinically and in a mouse model $(13,14)$.

A microRNA (miR) is a small sequence of 22 bases that binds to messenger RNA with a complementary sequence and suppresses it by inhibiting translation or by promoting degradation. miR mainly recognize seven bases on the target messenger 
RNA sequence, namely the 2nd-8th base on the 3 ' end (15). The preconditioning effects of anesthesia and ischemia on IRI are reportedly mediated via changes in miR $(16,17)$. However, the associations between miRs and preconditioning effects on the kidney have not been fully clarified.

The present study compared sevoflurane APC and IPC in a rat renal IRI model, verified the dynamics of miRs and researched target cell-survival pathways. A pathway analysis system was used to estimate the connection between the related genes affected by APC or IPC with canonical pathways from a database including a large number of molecular interactions and gene-to-phenotype studies. Rat IRI models have also been previously used to elucidate the known cell-death or cell-survival substances common to humans. The present study aimed to clarify the cell-survival pathways that are affected by sevoflurane APC and IPC, and to find commonalities and differences in these preconditioning effects using a rat IRI model.

\section{Materials and methods}

Ethics statement. The experimental protocols were approved by the Animal Research Committee at Nippon Medical School (Approval number: 29-008, Approval date: April 01, 2017). In addition, all the experimental protocols were performed in accordance with the ARRIVE guidelines.

Surgical preparation. A total of 28 male Wistar rats (age 10-11 weeks, weighing 336 $\pm 24 \mathrm{~g}$, obtained from Tokyo Laboratory Animals Science Co., Ltd.) were kept in cages at $26^{\circ} \mathrm{C}$ under a $14: 10 \mathrm{~h}$ light:dark cycle in a specific pathogen-free facility, with free access to food and water. Before the surgical procedure, anesthesia was induced with $2 \mathrm{mg} / \mathrm{kg}$ midazolam, $2.5 \mathrm{mg} / \mathrm{kg}$ butorphanol and $0.15 \mathrm{mg} / \mathrm{kg}$ medetomidine hydrochloride injected intraperitoneally, followed by injection of $0.6 \mathrm{mg} / \mathrm{kg} / \mathrm{h}$ midazolam, $0.8 \mathrm{mg} / \mathrm{kg} / \mathrm{h}$ butorphanol and $0.05 \mathrm{mg} / \mathrm{kg} / \mathrm{h}$ medetomidine hydrochloride to maintain sedation and analgesia. Additional $0.6 \mathrm{mg} / \mathrm{kg}$ midazolam, $0.8 \mathrm{mg} / \mathrm{kg}$ butorphanol and $0.05 \mathrm{mg} / \mathrm{kg}$ medetomidine hydrochloride were administered if the rats moved their limbs in response to pain. They were ventilated through a tracheal tube to maintain the partial pressure of carbon dioxide at $35-45 \mathrm{mmHg}$. Rectal temperature was maintained at $36.5-37.5^{\circ} \mathrm{C}$. Mean arterial pressure was monitored via the right femoral artery. To maintain fluid volume, a normal saline solution $(3 \mathrm{ml} / \mathrm{h})$ was continuously infused via the caudal vein. Under general anesthesia, the right kidney was resected via a median abdominal incision (18). The time from initiation of general anesthesia to resection of the right kidney was $40 \mathrm{~min}$. Next, the effects of IRI of the left kidney and the ameliorative effects of APC and IPC on IRI were evaluated. For this, the 28 rats were randomly allocated to four groups: i) Sham group (no left renal arteriovenous clamping); ii) IRI group (45 min of left renal arteriovenous clamping and $4 \mathrm{~h}$ of reperfusion); iii) APC group (exposure to one minimum alveolar concentration sevoflurane $2.2 \%$ for $15 \mathrm{~min}, 10 \mathrm{~min}$ before the IRI procedure) and iv) IPC group (three cycles of renal arteriovenous clamping for $2 \mathrm{~min}$ and reperfusion for $5 \mathrm{~min}$ immediately before the IRI procedure) (Fig. 1). To minimize technical noise, four randomly chosen rats, one from each group, underwent the surgical procedure at the same time. Accurate performance of the IRI procedure was confirmed by observation of loss and return of redness of the kidney. Reperfusion was considered successful when redness in the entire kidney returned immediately after arteriovenous release. At the end of the procedure, the left kidney tissue was sampled for analysis of extracted miR expression at the same time as blood was collected for measurement of serum creatinine as an indicator of renal IRI. The rats were subsequently sacrificed by exsanguination under general anesthesia.

Serum creatinine assay. Blood samples $(3 \mathrm{ml})$ were injected into a separating agent, centrifuged at $4^{\circ} \mathrm{C}$ and $3,000 \mathrm{x} \mathrm{g}$ for $15 \mathrm{~min}$, and the resultant supernatant was collected as serum (19). Serum creatinine levels were measured in a clinical laboratory using a clinical chemistry analyzer (JCA-BM6070; JEOL Ltd.) and the results were reported in $\mathrm{mg} / \mathrm{dl}$.

miR screening test. The left kidney tissue sample was permeated with RNA later solution ${ }^{\mathrm{TM}}$ (Applied Biosystems; Thermo Fisher Scientific, Inc.) and stored at $-80^{\circ} \mathrm{C}$. The tissue samples were sufficiently lysed in liquid nitrogen and total RNA was extracted using the mirVana miRNA Isolation Kit ${ }^{\mathrm{TM}}$ (Applied Biosystems; Thermo Fisher Scientific, Inc.) and assessed with the NanoDrop ND-1000 ${ }^{\mathrm{TM}}$ spectrophotometer (Thermo Fisher Scientific, Inc.) (17).

Quantitative measurement of miR expression. The rat renal cyclic DNA was reverse transcribed using a TaqMan ${ }^{\mathrm{TM}}$ MicroRNA Reverse Transcription kit (Applied Biosystems; Thermo Fisher Scientific, Inc.) and Megaplex ${ }^{\mathrm{TM}} \mathrm{RT}$ primers A and B (Applied Biosystems; Thermo Fisher Scientific, Inc.), according to the following temperature protocol: 40 cycles of $16^{\circ} \mathrm{C}$ for $2 \mathrm{~min}, 42^{\circ} \mathrm{C}$ for $1 \mathrm{~min}$ and $50^{\circ} \mathrm{C}$ for $1 \mathrm{sec}$, followed by $85^{\circ} \mathrm{C}$ for $5 \mathrm{~min}$, and storage at $4^{\circ} \mathrm{C}$. Subsequently, 373 specific kinds of well-known miRs derived from rats were amplified and measured with the TaqMan Low Density Array ${ }^{\mathrm{TM}}$ (Applied Biosystems; Thermo Fisher Scientific, Inc.) in the 7900 HT Fast Real-Time polymerase chain reaction System ${ }^{\mathrm{TM}}$ (Applied Biosystems; Thermo Fisher Scientific, Inc.) using a comparative quantification method.

The results of reverse transcription-quantitative (RT-q)PCR were analyzed with Data Assist software version 2.0 (Applied Biosystems; Thermo Fisher Scientific, Inc.) as follows: i) the control gene was set as Y1, which has the highest stable value calculated among the candidates U6, U87 and Y1; ii) the $\Delta \mathrm{Cq}$ value of each miR was obtained by comparing the number of amplifications until it exceeded the automatic threshold value with that of $\mathrm{Y} 1$; iii) the $\Delta \Delta \mathrm{Cq}$ value of each $\mathrm{miR}$ in each group was obtained by subtracting the mean $\Delta \mathrm{Cq}$ value in the sham group from that in each group and the miR expression value, as the Fold-Change $\left(\right.$ F.C. $=2^{-\Delta \Delta C q}$ ) value, was obtained; and iv) the adjusted F.C. value of each miR in each group was calculated by dividing its F.C. value by the mean F.C. value of the sham group (20).

Search for the target pathway using Ingenuity Pathway Analysis ${ }^{T M}$. The list of differentially expressed miRs was analyzed using Ingenuity Pathway Analysis ${ }^{\text {TM }}$ (IPA; Qiagen, Inc.) to estimate related genes and canonical pathways using the 'core analysis' function, and mapped in the 
Experimental protocols

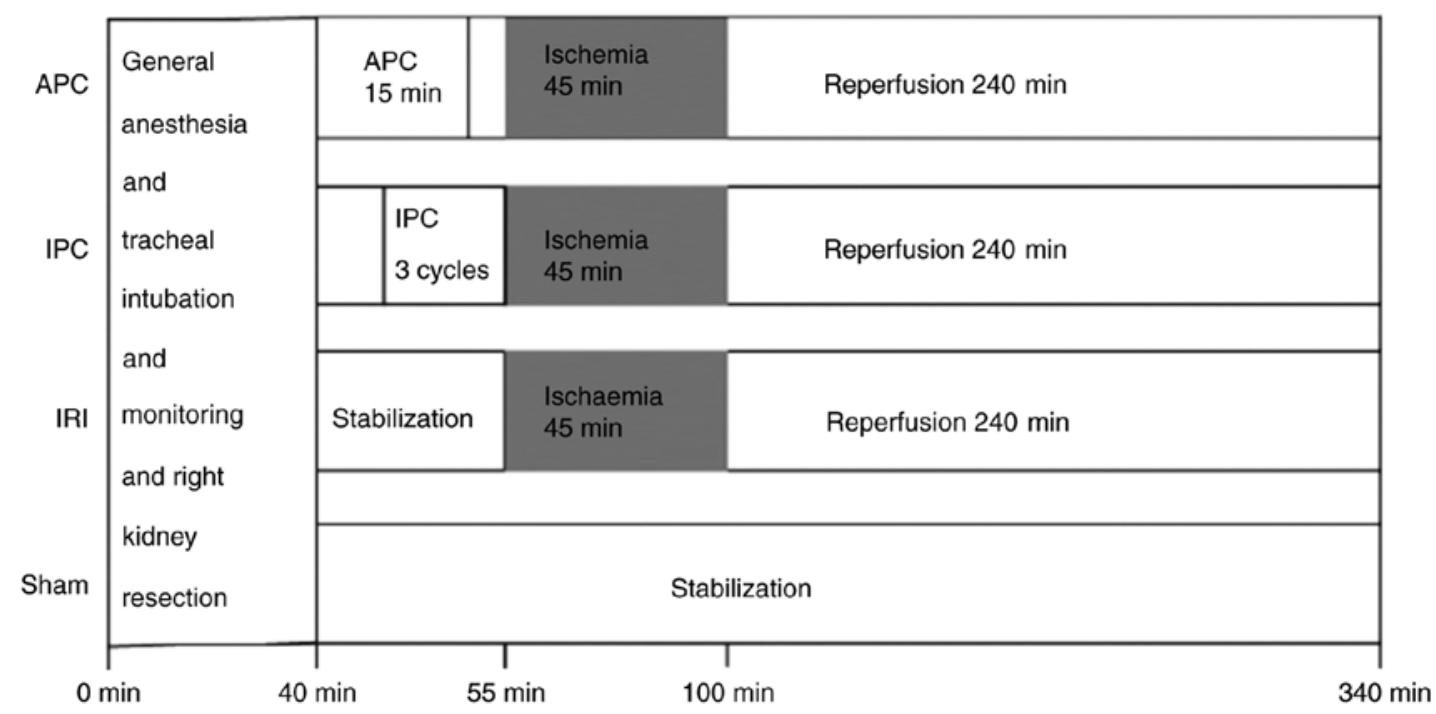

Figure 1. Experimental protocols in the four groups. The rats were divided into four groups. All the rats underwent right nephrectomy within 40 min after the start of the protocol. APC, anesthetic preconditioning; IPC, ischemic preconditioning; IRI, ischemia reperfusion injury.

predicted network based on a database including molecular interactions and gene-to-phenotype studies from over 200,000 peer-reviewed scientific articles. This analysis was performed to narrow down the canonical pathways affected by the miRs assessed in this experiment.

Measurement of activation of the target pathway using western blot analysis. Western blot analysis was performed to verify activation of the target substances in cell-survival pathways that were predicted by IPA ${ }^{\mathrm{TM}}$ to be related to the experimentally changed miRs. Total renal proteins were extracted using T-PER Tissue Protein Extraction Reagent ${ }^{\mathrm{TM}}$ (Pierce; Thermo Fisher Scientific, Inc.) with Halt Protease Inhibitor Cocktail EDTA-Free ${ }^{\mathrm{TM}}$ (Pierce; Thermo Fisher Scientific, Inc.), and quantified with Quick Start ${ }^{\mathrm{TM}}$ Bradford Reagent (Bio-rad Laboratories, Inc.). A total of $40 \mu \mathrm{g}$ protein per lane were applied into Mini-PROTEAN TGX Stain-Free Gels $^{\mathrm{TM}}$ (4-15\%; Bio-rad Laboratories, Inc.; cat. no. 456-8084) soaked in Tris/Glycine/SDS Buffer (Bio-Rad Laboratories, Inc.) and transferred onto a polyvinylidene fluoride membrane. Followed by incubation overnight at $4^{\circ} \mathrm{C}$ in TBS containing $0.1 \%$ Tween-20 (Bio-Rad Laboratories, Inc.) with $5 \%$ bovine serum albumin (Sigma-Aldrich; Merck KGaA) and several antibodies, including anti-phosphatase and tensin homologue deleted from chromosome 10 (PTEN) (A2B1; Mouse monoclonal antibody; 1:200; Santa Cruz Biotechnology, Inc.; cat. no. SC-7974), anti-protein kinase B (Akt) (pan) (C67E7; Rabbit monoclonal antibody; 1:1,000; Cell Signaling Technology, Inc.; cat. no. 4691S), anti-phosphorylated Akt (pAkt) (Ser473) (XP Rabbit monoclonal antibody; 1:1,000; Cell Signaling Technology, Inc.; cat. no. 4060S) and anti- $\beta$-actin (C4) (Mouse monoclonal antibody; 1:200; Santa Cruz Biotechnology, Inc.; cat. no. SC-47778), respectively after suppressing non-singular bands using 5\% Amersham ECL Blocking Agent ${ }^{\mathrm{TM}}$ (GE Healthcare UK Ltd.). The protein bands of the four different groups were arranged in a row on the same membrane ( $n=7$ for each antibody used). Protein bands were visualized with chemiluminescence using a goat polyclonal second antibody to rabbit IgG HRP (1:20,000; Abcam; cat. no. 643005) or goat polyclonal second antibody to mouse IgG1 HRP (1:20,000; Cosmo Bio Co., Ltd.; cat. no. A90-105P), followed by Amersham ECL Prime Western blotting detection reagent ${ }^{\mathrm{TM}}$ (GE Healthcare UK Ltd.) in an ImageQuant LAS 4000 mini biomolecular imager (GE Healthcare). The intensity of protein fragments was quantified with ImageQuant TL ver. 8.1 (GE Healthcare). Since the quantified signal values involved background differences between the seven membranes used, the F.C. value of each target protein was calculated as the ratio to $\beta$-actin. The adjusted F.C. value was obtained by correction using a ratio of $\beta$-actin signal value in the sham group on the same membrane to the average of that on all the membranes used.

Statistical analysis. All the 28 kidneys sampled were analyzed. Vital signs, serum creatinine values and adjusted F.C. values of the miRs and the target proteins are expressed as mean \pm standard deviation. Sample size was calculated for the serum creatinine value and adjusted F.C. values of the target proteins and miRs. A sample size of seven per group has an effect size of 0.8 with the power one-way analysis of variance (ANOVA) test using a significance value of 0.05 and a power of 0.9. All the $\Delta \Delta \mathrm{Cq}$ values of miRs and vital signs and serum creatinine values were normally distributed and inter-group comparisons were made using Tukey's test. To sufficiently narrow down the miRs to be analyzed, a one way ANOVA was performed on the apparently differentially expressed miRs following APC or IPC, of which the adjusted F.C. value in APC or IPC satisfied the following conditions: i) adjusted F.C. $\geq 1.2$ and not less than four times that in the IRI group; and ii) adjusted F.C. $\leq 0.8$, and not more than quarter that in the IRI group. Multiple tests were corrected with the Storey's method for turning the list of P-values into q-values, which measures the false discovery rate. Then, the final intergroup difference determination was performed by multiple comparisons with Tukey's tests, which 
Table I. Vital signs of the rats in each group during the experimental protocol.

A, Heart rate of the rats in each group during the experimental protocol

\begin{tabular}{|c|c|c|c|c|}
\hline Group & Baseline, $\min ^{-1}$ & $\begin{array}{l}\text { Ischemia for } \\
45 \mathrm{~min}, \mathrm{~min}^{-1}\end{array}$ & $\begin{array}{l}\text { Reperfusion for } \\
3 \mathrm{~min}, \mathrm{~min}^{-1}\end{array}$ & $\begin{array}{l}\text { Reperfusion } \\
\text { for } 4 \mathrm{~h}, \mathrm{~min}^{-1}\end{array}$ \\
\hline Anesthetic preconditioning & $215 \pm 14$ & $225 \pm 19$ & $227 \pm 25$ & $218 \pm 24$ \\
\hline Ischemic preconditioning & $252 \pm 65$ & $274 \pm 41$ & $284 \pm 45$ & $259 \pm 31$ \\
\hline Ischemia & $209 \pm 16$ & $222 \pm 20$ & $207 \pm 9$ & $238 \pm 31$ \\
\hline Sham & $219 \pm 9$ & $211 \pm 10$ & $214 \pm 9$ & $205 \pm 9$ \\
\hline
\end{tabular}

$\mathrm{B}$, Mean atrial pressure of the rats in each group during the experimental protocol

\begin{tabular}{|c|c|c|c|c|}
\hline Group & Baseline, $\min ^{-1}$ & $\begin{array}{l}\text { Ischemia for } \\
45 \mathrm{~min}, \mathrm{~min}^{-1}\end{array}$ & $\begin{array}{l}\text { Reperfusion for } \\
3 \mathrm{~min}, \mathrm{~min}^{-1}\end{array}$ & $\begin{array}{l}\text { Reperfusion } \\
\text { for } 4 \mathrm{~h}, \mathrm{~min}^{-1}\end{array}$ \\
\hline Anesthetic preconditioning & $86 \pm 18$ & $87 \pm 24$ & $81 \pm 14$ & $96 \pm 26$ \\
\hline Ischemic preconditioning & $100 \pm 18$ & $104 \pm 17$ & $105 \pm 12$ & $93 \pm 15$ \\
\hline Ischemia & $103 \pm 20$ & $91 \pm 16$ & $100 \pm 26$ & $81 \pm 17$ \\
\hline Sham & $94 \pm 14$ & $88 \pm 11$ & $87 \pm 10$ & $75 \pm 9$ \\
\hline
\end{tabular}

were performed on the miRs having significant inter-group differences $(\mathrm{P}<0.05, \mathrm{q}<0.5)$.

Since the expression values of target proteins were not normally distributed by the Shapiro-Wilk test $(\mathrm{P}=0.0002)$, the Kruskal Wallis test followed by Bonferroni's multiple comparison test was performed for each group. $\mathrm{R}$ version 3.4.2 was used in all statistical tests, which is a language and environment for statistical computing (http://cran.r-project.org/).

\section{Results}

Hemodynamics during surgery. All the 28 rats allocated to the four groups (APC, IPC, IRI and Sham group) survived during the surgical procedures. There were no significant differences in heart rate and mean atrial pressure at the four time points of the beginning of the experimental protocol (baseline), immediately before reperfusion (ischemia for $45 \mathrm{~min}$ ), $3 \mathrm{~min}$ after ischemia release (reperfusion for $3 \mathrm{~min}$ ), and just before removal of the left kidney $4 \mathrm{~h}$ after reperfusion (finish) between the four groups (Table I).

Serum creatinine levels. Serum creatinine values, as an indicator of IRI, were significantly higher in the IRI group $(0.96 \pm 0.13 \mathrm{mg} / \mathrm{dl})$ than the sham group $(0.54 \pm 0.06 \mathrm{mg} / \mathrm{dl}$; $\mathrm{P}<0.001)$. Serum creatinine values in APC $(0.71 \pm 0.08 \mathrm{mg} / \mathrm{dl})$ and IPC $(0.73 \pm 0.10 \mathrm{mg} / \mathrm{dl})$ groups were higher than those in the sham group (APC, $\mathrm{P}=0.029$; $\mathrm{IPC}, \mathrm{P}=0.011$ ), although they were significantly lower than those in the IRI group (APC, $\mathrm{P}<0.001$; IPC, $\mathrm{P}=0.002)$. There were no significant differences between the APC and IPC groups (Fig. 2).

miR screening tests and analyses. Out of the 373 types of well-known miRs derived from rats, one-way ANOVA was performed for 125 kinds of miRs that fulfilled either condition 1 or 2, as previously described. Tukey's test was performed

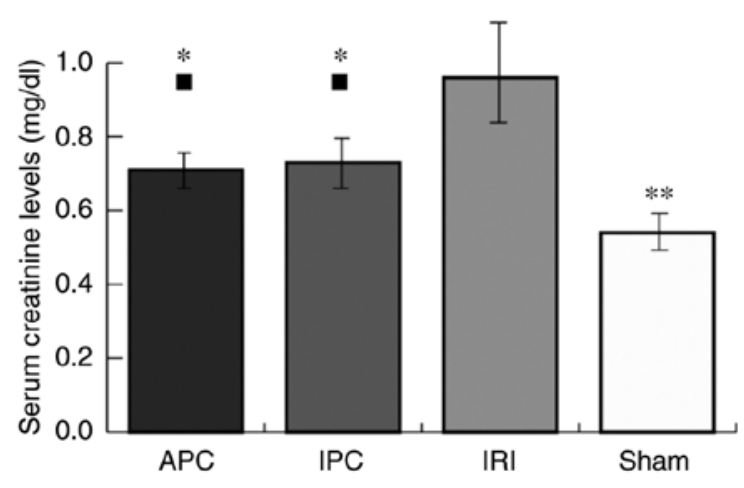

Figure 2. Serum creatinine levels. Serum creatinine levels were expressed as the mean \pm standard deviation $(\mathrm{mg} / \mathrm{dl}) .{ }^{*} \mathrm{P}<0.05$ vs. the Sham group. ${ }^{*} \mathrm{P}<0.05$ and ${ }^{* *} \mathrm{P}<0.01$ vs. the IRI group. APC, anesthetic preconditioning; IPC, ischemic preconditioning; IRI, ischemia reperfusion injury.

on 10 miRs identified by one-way ANOVA corrected with Storey's method as having significant differences in adjusted F.C. values among the four groups $(\mathrm{P}<0.05, \mathrm{q}<0.5)$.

As a result, significant differences in seven miRs were noted between the APC and IRI group or between the IPC and IRI group $(\mathrm{P}<0.05$; Table II). The dataset of the results of RT-qPCR are available in a data repository (doi: 10.17632/dmngjht9sg.1).

Search for the predicted networks between specific miRs and the cell-survival pathway. IPA analysis of the seven miRs, which were differentially expressed following APC or IPC procedures as previously described, showed that miR-17-3p, miR-19a/b, miR-34a and miR-27a might be involved in the cell-survival pathway associated with the specific substance 'Akt' or 'TP53' (Fig. 3). RT-qPCR in the current study showed that sevoflurane APC promoted miR-17-3p, but suppressed miR-27a, and that IPC promoted miR-19a and miR-34a 
Table II. Significant changes in miRs following preconditioning procedures.

A, Significantly different miRs between anesthetic preconditioning and ischemia groups

\begin{tabular}{lcccc}
\hline & \multicolumn{2}{c}{ Adjusted fold-change } & & \\
\cline { 2 - 3 } $\operatorname{miR}$ & APC & IRI & APC/IRI & P-value \\
\hline miR-17-3p & $2.14 \pm 1.12$ & $0.20 \pm 0.076$ & 10.9 & $0.016(<0.05)$ \\
miR-27a & $0.74 \pm 0.22$ & $4.56 \pm 3.15$ & 0.16 & $0.005(<0.01)$ \\
\hline
\end{tabular}

B, Significantly different miRs between ischemic preconditioning and ischemia groups

\begin{tabular}{lcccc}
\hline & \multicolumn{2}{c}{ Adjusted fold-change } & & \\
\cline { 2 - 3 } miR & IPC & IRI & IPC/IRI & P-value \\
\hline miR-125a & $3.70 \pm 2.59$ & $0.72 \pm 0.65$ & 5.11 & $0.034(<0.05)$ \\
miR-19a & $1.97 \pm 0.77$ & $0.48 \pm 0.36$ & 4.14 & $0.003(<0.005)$ \\
miR-34a & $1.95 \pm 1.47$ & $0.11 \pm 0.025$ & 17.4 & $0.040(<0.05)$ \\
miR-872 & $2.35 \pm 0.42$ & $0.33 \pm 0.21$ & 7.17 & $<0.001$ \\
miR-450a & $9.02 \pm 8.77$ & $0.32 \pm 0.19$ & 28.2 & $0.035(<0.05)$ \\
\hline
\end{tabular}

APC, anesthetic preconditioning; IPC, ischemic preconditioning; IRI, ischemia reperfusion injury; miR, microRNA.

(Table II). Although IPC also promoted miR-125a, miR-872 and miR-450a in the current study, IPA analysis did not identify any association between the cell-survival pathway and these miRs.

The predicted functional network presented by IPA ${ }^{\mathrm{TM}}$ and a further literature search suggested that the sevoflurane APC and IPC models might block PTEN and increase pAkt (Fig. 4). Western blot analysis indicated decreased PTEN and increased pAkt in the APC and IPC groups.

IPA analysis suggested that sevoflurane APC and IPC affect Akt phosphorylation. Western blot analysis also showed that the adjusted F.C. value of pAkt was increased in both the APC and IPC groups compared with the IRI group $(\mathrm{P}=0.013$, $\mathrm{P}=0.013$ ). Furthermore, the adjusted F.C. value of PTEN was decreased in both APC and IPC groups compared with the IRI group ( $\mathrm{P}=0.013, \mathrm{P}=0.013$ ) (Fig. 5; the full-length blots in the main paper are displayed in Fig. S1).

\section{Discussion}

The present study confirmed that sevoflurane APC and IPC have reno-protective effects and affect specific miRs that were predicted by IPA in the current study to regulate reno-protection. However, there are few comparative reports of sevoflurane APC and IPC in terms of the magnitude of reno-protective effects and the dynamics of miRs regulating the reno-protection. This study revealed that sevoflurane APC and IPC equally ameliorated renal IRI, as indicated by serum creatinine values, equally suppressed PTEN, and equally increased pAkt, although the effect was brought about via different miRs.

The current study predicted that PTEN and pAkt are important signaling agents involved in cell-survival, that are regulated by the miRs differentially expressed after both sevoflurane APC and IPC, using IPA and a further detailed literature search. As revealed by the literature search, miR-17-3p that increases with APC and miR-19a that increases with IPC, respectively, block PTEN directly and increase pAkt via the PTEN/phosphoinositide 3-kinase (PI3K)/Akt pathway, whereas miR-27a, which decreases with APC, blocks PI3K directly and decreases pAkt (21-23), along with suppression of PTEN, although via different microRNAs, followed by equal increases in pAkt.

Preconditioning effects have been reported in clinical and animal research. In studies on sevoflurane APC during cardiac surgery, acute renal IRI caused by cardiac arrest could be ameliorated by administering sevoflurane immediately before cardiac arrest (14). The sevoflurane APC effect on mice kidneys was also reported in vivo (13). As far as is known, although the efficacy of renal IPC has not yet been shown to be clinically significant, unlike remote IPC for the kidney, renal IPC in mice reportedly suppresses the cell-death caused by subsequent IRI $(8,9)$.

Multiple cell-survival pathways triggered by IRI have been previously reported, based on the background of IRI and the effects of preconditioning (24-26). The PTEN/PI3K/Akt pathway is an important intracellular signaling pathway in the regulation of the cell cycle. PTEN directly blocks downstream activity of the cell-survival PI3K/Akt pathway (27). Akt is known to have three isoforms, with each isoform playing several specific roles in the signaling pathways, such as cell survival, cell proliferation, neovascularization and glucose metabolism (28). Akt promotes cell-survival by promoting several anti-apoptotic pathways $(21,29)$. Activated PI3K alters the conformation of Akt, to allow phosphoinositide-dependent kinase-1 to phosphorylate Akt, reportedly (30). Activation 


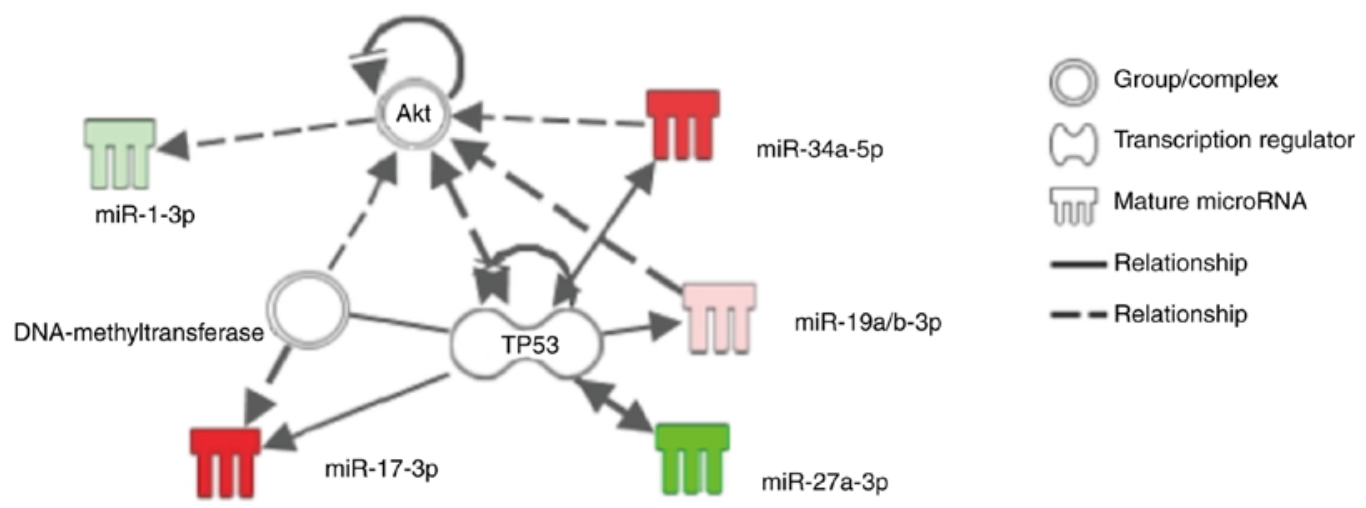

Figure 3. Predicted functional network between the differentially expressed microRNAs following APC or IPC and the related cell-survival pathway using IPA. This figure represents a simplified network estimated by IPA drawn using an IPA drawing tool. Some of the microRNAs affected by APC or IPC, including miR-17-3p, miR-19a, miR-27a and miR-34a, were estimated to be associated with the substances 'TP53' and 'Akt'. Solid lines represent direct interactions and dashed lines represent indirect interactions. APC, anesthetic preconditioning; IPC, ischemic preconditioning; miR, microRNA; Akt, protein kinase B; IPA, ingenuity pathway analysis.

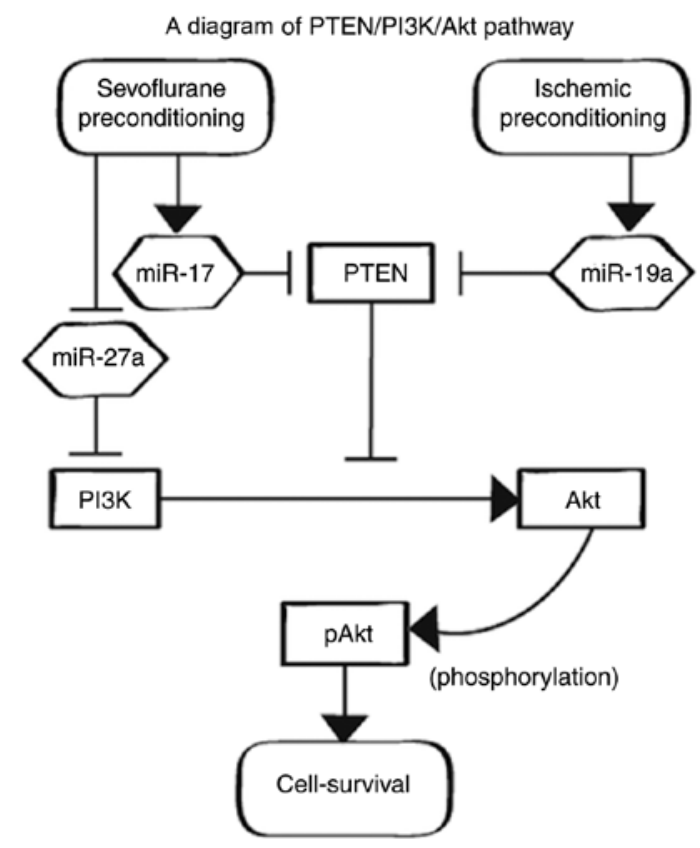

Figure 4. PTEN/PI3K/Akt pathway. pAkt plays an important role in cell survival. PTEN downregulates the PI3K/Akt signaling pathway, decreasing pAkt. PTEN is downregulated by both miR-17 and miR-19a, which are promoted by APC and IPC, respectively. PI3K is downregulated by miR-27a APC, anesthetic preconditioning; IPC, ischemic preconditioning; pAkt, phosphorylated protein kinase B; PTEN, phosphatase and tensin homologue deleted from chromosome 10; PI3K, phosphoinositide 3-kinase.

of the Akt pathway reportedly reduces hypoxia-induced cell apoptosis, such that overexpression of PTEN causes cell-death, while suppression of PTEN has a cell-survival effect $(27,29)$

IPC reportedly increases pAkt via the PTEN/PI3K/Akt pathway, resulting in suppression of rat myocardial cell apoptosis $(24,31)$. In addition, IPC of mice kidneys suppressed the cell-death caused by subsequent IRI, increasing the anti-apoptotic activity of pAkt (9). Sevoflurane APC reportedly ameliorates rat myocardial IRI by regulating the PTEN/PI3K/Akt pathway, similar to the rat IPC model (32). In the current study, it was also found that sevoflurane

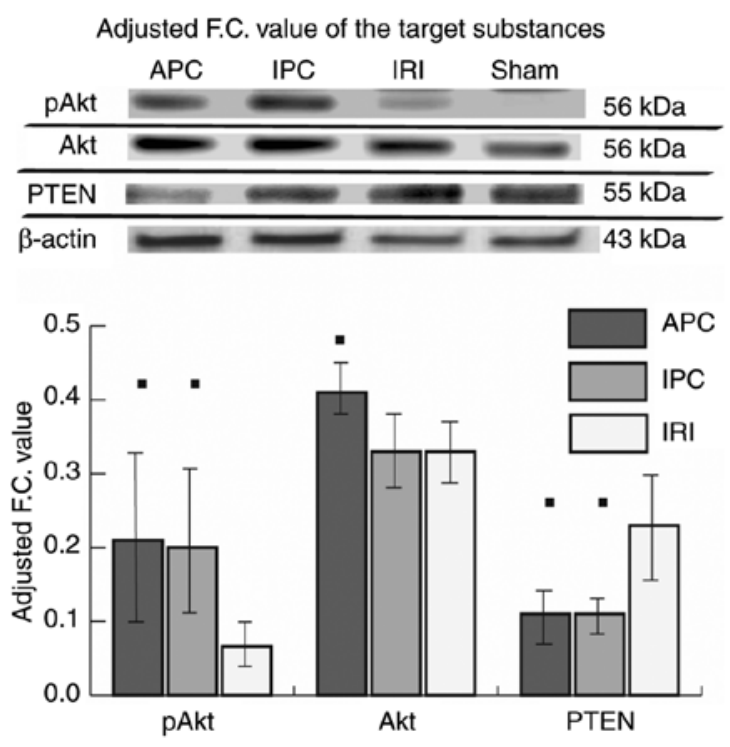

Figure 5. Expression levels of PTEN, pAkt and Akt. The protein bands of rats in the four groups were arranged in a row on the same membrane $(n=7$ for each antibody used). Since the quantified signal values have different backgrounds between all the membranes used, the F.C. value of pAkt, Akt or PTEN in each group was calculated as the ratio to $\beta$-actin and the adjusted F.C. value was obtained by correction using the ratio of the $\beta$-actin signal value in the sham group on the same membrane to the average of that on all the membranes used and was expressed as the mean \pm standard deviation. The $\mathrm{y}$-axis of the graph represents the adjusted F.C. value. Inter-group comparisons were made using Bonferroni's multiple comparison test. ${ }^{-} \mathrm{P}<0.05$ vs. the IRI group. APC, anesthetic preconditioning; IPC, ischemic preconditioning; IRI, ischemia reperfusion injury; pAkt, phosphorylated protein kinase B; F.C., fold-change; PTEN, phosphatase and tensin homologue deleted from chromosome 10 .

APC and IPC ameliorate renal IRI in rats, probably via the PTEN/PI3K/Akt pathway.

Genetic changes, including specific miR changes, were reported to suppress IRI by affecting specific cell-survival pathways (33-35). The PTEN/PI3K/Akt pathway is also regulated by multiple miRs $(23,36,37)$. The present study found that sevoflurane APC promoted miR-17-3p expression, whereas IPC promoted miR-19a expression. There was an important commonality between sevoflurane APC and 
IPC effects. The miR-17/92 cluster consists of seven miRs (miR-17-3p, miR-17-5p, miR-18, miR-19a, miR-20a, miR-19b-1 and miR-92a-1) located close to the chromosome 13 (13q31.3) region and produced from a common host RNA (34). Reportedly, both miR-17 and miR-19a directly block PTEN, because multiple binding sites for such miRs as miR-19a/b, miR-17 and miR-20a are conserved in the 3'-untranslated region of PTEN messenger RNA $(36,37)$. Other studies also reported that miR-17-3p directly blocks PTEN and increases pAkt via the PTEN/PI3K/Akt pathway, similar to miR-19a that blocks PTEN directly and increases pAkt $(21,22,38)$. Thus, sevoflurane APC and IPC might equally suppress PTEN because of the similarity in their affected miRs.

The effect of PTEN inhibition produced by both types of preconditioning might affect a wide range of pathways involving various protein kinases that regulate cell-survival and not only the PI3K/Akt pathway. PTEN reportedly regulates apoptosis via phosphorylated mitogen-activated protein kinase-1/2/extracellular signal-regulated kinase-1/2 signaling and caspase-3/B-cell lymphoma-2 signaling (39).

Although sevoflurane APC and IPC might affect the common PTEN/PI3K/Akt signaling pathway, there are differences in the directly affected miRs. The current study found that sevoflurane APC suppresses miR-27a expression. In nucleus pulposus cells, miR-27a directly blocks PI3K, but not PTEN (23). The authors anticipated that sevoflurane APC might affect multiple signaling stages in the $\mathrm{PTEN} / \mathrm{PI} 3 \mathrm{~K} / \mathrm{Akt}$ pathway, resulting in the increase in pAkt in renal cells. Directly promoting PI3K might also activate other anti-apoptotic pathways dependent on PI3K. Reportedly, a model of reperfusion 3 days after permanent middle cerebral artery occlusion resulted in activation of the fibroblast growth factor $21 /$ fibroblast growth factor receptor $1 / \mathrm{PI} 3 \mathrm{~K} /$ caspase-3 pathway and reduced neuronal apoptosis (40). Sevoflurane APC might thus reduce caspase- 3 by its close involvement in the apoptotic process via the PI3K/Caspase-3 pathway.

The present study found that IPC promotes miR-34a expression. It was previously reported that miR-34a expression is promoted in renal cells with overexpression of the protein cMYC, which directly binds and activates the miR-17/92 cluster (41). Therefore, the authors of the current study anticipated that IPC might increase cMYC, activating the miR-17/92 cluster and resulting in miR-34a overexpression. IPC might thus increase pAkt not only by suppressing PTEN, but also by activating the cMYC/miR-17/92 cluster/PTEN/PI3K/Akt pathway. In the future, further research is necessary to elucidate detailed differences between sevoflurane APC and IPC effects.

The present study showed that the sevoflurane APC effect reported so far is also found in the rat kidney. Furthermore, the current study found that sevoflurane APC has equivalent reno-protective effects compared with IPC, as indicated by both serum creatinine levels and those of the organ protective pAkt, and partially compared with its genetic mechanism in renal cells for the first time.

The present study has some limitations. The first limitation is that the reperfusion time after renal ischemia was set as $4 \mathrm{~h}$. It is necessary to evaluate the optimal time at which miR changes become most noticeable for accurate evaluation of the preconditioning effects. The second limitation is that serum creatinine values were not measured before surgery. Since the volume of blood required for measurement is more than $1 \mathrm{ml}$, preoperative blood sampling might cause organ ischemia. However, since the weights of all the rats randomly allocated to the four groups were similar and the environmental factors were also the same, baseline serum creatinine values are assumed to have been minimally different. The third limitation is that, although the main outcome in the present study was to prove a decrease in acute renal dysfunction with APC and IPC, it is necessary to prove the anti-apoptotic effect of APC and IPC histologically using renal tissue sampling in the future. Finally, although the current protocol suggested that the reno-protective effects of APC and IPC were related to regulation of specific miRs, as seen with RT-qPCR, experiments to identify the pathways involved using miR inhibitors, PTEN inhibitor and PI3K inhibitor are necessary in the future.

The present study concluded that sevoflurane APC and IPC had equivalent reno-protective effects in rats, although by different miR dynamics. However, it was estimated that both the preconditioning procedures resulted in an increase in pAkt and a decrease in PTEN. The current study estimated that these preconditioning effects might occur via the common cell-survival PTEN/PI3K/Akt pathway using IPA ${ }^{\mathrm{TM}}$ analysis and further literature search. The current study results might be useful for examining methods for alleviating renal IRI using general anesthesia and surgical procedures. Further study will be necessary to elucidate the differences in reno-protective effects induced by changing the type or amount of general anesthetic drugs used.

\section{Acknowledgements}

The authors wish to thank Ms. Miyuki Takatori and Ms. Kiyomi Kikukawa (Collaborative Research Center Laboratory for Clinical Research, Nippon Medical School, Tokyo, Japan) for their help as scientific advisers. The authors thank the laboratory technicians at the Department of Inspection, Nippon Medical School, for their help in testing the blood samples.

\section{Funding}

The present study was supported in part by grants from the Japanese Ministry of Education, named MEXT KAKENHI (grant nos. JP15K10525 and JP18K08870).

\section{Availability of data and materials}

The dataset of quantitative PCR of the current study was published and is available in the Mendeley Data repository (doi: 10.17632/dmngjht9sg.1).

\section{Authors' contributions}

MY contributed to conceptualizing and designing the study, data collection, analysis and interpretation of the data, and drafting of the manuscript. TM contributed to conceptualizing and designing the study, data collection, and critical revision of the manuscript. MI participated in designing the study, analysis of data and critical revision of the manuscript. AS 
contributed to conceptualizing and designing the study, critical revision of the manuscript, and was the principal investigator and had overall responsibility for the trial. All authors read and approved the final manuscript.

\section{Ethics approval and consent to participate}

The experimental protocols were approved by the Animal Research Committee at Nippon Medical School, Tokyo, Japan (Approval number: 29-008, Approval date: April 01, 2017). In addition, all the experimental protocols were performed in accordance with ARRIVE guidelines.

\section{Patient consent for publication}

Not applicable.

\section{Competing interests}

The authors declare that they have no competing interests.

\section{References}

1. Debout A, Foucher Y, Trébern-Launay K, Legendre C, Kreis H Mourad G, Garrigue V, Morelon E, Buron F, Rostaing L, et al: Each additional hour of cold ischemia time significantly increases the risk of graft failure and mortality following renal transplantation. Kidney Int 87: 343-349, 2015.

2. Girerd S, Frimat L, Ducloux D, Le Meur Y, Mariat C, Moulin B, Mousson C, Reiu P, Dali-Youcef N, Merckle L, et al: EPURE transplant (eplerenone in patients undergoing renal transplant) study: Study protocol for a randomized controlled trial. Trials 19: $595,2018$.

3. Tonelli M, Wiebe N, Knoll G, Bello A, Browne S, Jadhav D, Klarenbach $S$ and Gill J: Systematic review: Kidney transplantation compared with dialysis in clinically relevant outcomes. Am J Transplant 11: 2093-2109, 2011.

4. Lannemyr L, Bragadottir G, Hjärpe A, Redfors B and Ricksten SE: Impact of cardiopulmonary bypass flow on renal oxygenation in patients undergoing cardiac surgery. Ann Thorac Surg 107: 505-511, 2019.

5. Nadim MK, Forni LG, Bihorac A, Hobson C, Koyner JL, Shaw A, Arnaoutakis GJ, Ding X, Engelman DT, Gasparovic H, et al: Cardiac and vascular surgery-associated acute kidney injury: The 20th international consensus conference of the ADQI (acute disease quality initiative) group. J Am Heart Assoc 7: e008834 2018.

6. Eltzschig HK and Eckle T: Ischemia and reperfusion- from mechanism to translation. Nat Med 17: 1391-1401, 2011.

7. Eltzschig HK: Targeting hypoxia-induced inflammation. Anesthesiology 114: 239-242, 2011.

8. Choi HS, Hwang JK, Kim JG, Hwang HS, Lee SJ, Chang YK, Kim JI and Moon IS: The optimal duration of ischemic preconditioning for renal ischemia-reperfusion injury in mice. Ann Surg Treat Res 93: 209-216, 2017.

9. Jang HS, Kim J, Kim KY, Kim JI, Cho MH and Park KM: Previous ischemia and reperfusion injury results in resistance of the kidney against subsequent ischemia and reperfusion insult in mice; a role for the Akt signal pathway. Nephrol Dial Transplant 27: 3762-3770, 2012.

10. Liang Y, Li Z, Mo N, Li M, Zhuang Z, Wang J, Wang Y and Guo $X$ : Isoflurane preconditioning ameliorates renal ischemia-reperfusion injury through antiinflammatory and antiapoptotic actions in rats. Biol Pharm Bull 37: 1599-1605, 2014.

11. Su MW, Chang SS, Chen CH, Hung CC, Chang SW, Tsai YC and Lam CF: Preconditioning renoprotective effect of isoflurane in a rat model of virtual renal transplant. J Surg Res 189: 135-142, 2014.

12. Lempiäinen J, Finckenberg P, Mervaala EE, Storvik M, Lindstedt K, Levijoki J and Mervaala EM: Dexmedetomidine preconditioning ameliorates kidney ischemia-reperfusion injury. Pharmacol Res Perspect 2: e00045, 2014.
13. Lee HT, Chen SW, Doetschman TC, Deng C, D'Agati VD and Kim M: Sevoflurane protects against renal ischemia and reperfusion injury in mice via the transforming growth factor-beta1 pathway. Am J Physiol Renal Physiol 295: F128-F136, 2008.

14. Julier K, da Silva R, Garcia C, Bestmann L, Frascarolo P, Zollinger A, ChassotPG, SchmidER, Turina MI, von Segesser LK, et al: Preconditioning by sevoflurane decreases biochemical markers for myocardial and renal dysfunction in coronary artery bypass graft surgery: A double-blinded, placebo-controlled, multicenter study. Anesthesiology 98: 1315-1327, 2003.

15. Bartel DP: MicroRNAs: Genomics, biogenesis, mechanism, and function. Cell 116: 281-297, 2004.

16. Godwin JG, Ge X, Stephan K, Jurisch A, Tullius SG and Iacomini J: Identification of a microRNA signature of renal ischemia reperfusion injury. Proc Natl Acad Sci USA 107: 14339-14344, 2010.

17. Morita T, Ishikawa M and Sakamoto A: Identical microRNAs regulate liver protection during anaesthetic and ischemic preconditioning in rats: An animal study. PLoS One 10: e0125866, 2015.

18. Takayama J, Takaoka M and Matsumura Y: Acute and chronic renal failure model in rats and mice. Nihon Yakurigaku Zasshi 131: 37-42, 2008 (In Japanese).

19. Liu L, Pang XL, Shang WJ, Xie HC, Wang JX and Feng GW: Over-expressed microRNA-181a reduces glomerular sclerosis and renal tubular epithelial injury in rats with chronic kidney disease via down-regulation of the TLR/NF- $\kappa \mathrm{B}$ pathway by binding to CRY1. Mol Med 24: 49, 2018.

20. Ishikawa M, Tanaka S, Arai M, Genda Y and Sakamoto A: Differences in microRNA changes of healthy rat liver between sevoflurane and propofol anesthesia. Anesthesiology 117: 1245-1252, 2012

21. Luan Y, Chen M and Zhou L: miR-17 targets PTEN and facilitates glial scar formation after spinal cord injuries via the PI3K/Akt/mTOR pathway. Brain Res Bull 128: 68-75, 2017.

22. Sun G, Lu Y, Li Y, Mao J, Zhang J, Jin Y, Sun Y, Liu L and Li L: miR-19a protects cardiomyocytes from hypoxia/reoxygenation-induced apoptosis via PTEN/PI3K/p-Akt pathway. Biosci Rep 37: BSR20170899, 2017.

23. Liu G, Cao P, Chen H, Yuan W, Wang J and Tang X: miR-27a regulates apoptosis in nucleus pulposus cells by targeting PI3K. PLoS One 8: e75251, 2013.

24. Han J, Xuan JL, Hu HR and Chen ZW: Protective effect against myocardial ischemia reperfusion injuries induced by hyperoside preconditioning and its relationship with PI3K/Akt signaling pathway in rats. Zhongguo Zhong Yao Za Zhi 40: 118-123, 2015 (In Chinese).

25. Wu J, Yu J, Xie P, Maimaitili Y, Wang J, Yang L, Ma H, Zhang X, Yang Y and Zheng H: Sevoflurane postconditioning protects the myocardium against ischemia/reperfusion injury via activation of the JAK2-STAT3 pathway. PeerJ 5: e3196, 2017

26. Liu C, Liu Y, Shen Z, Mial L, Zhang K, Wang F and Li Y: Sevoflurane preconditioning reduces intestinal ischemia-reperfusion injury: Role of protein kinase $\mathrm{C}$ and mitochondrial ATP-sensitive potassium channel. PLoS One 10: e0141426, 2015.

27. Sun G, Zhou Y, Li H, Guo Y, Shan J, Xia M, Li Y, Li S, Long D and Feng L: Over-expression of microRNA-494 up-regulates hypoxia-inducible factor-1 alpha expression via PI3K/Akt pathway and protects against hypoxia-induced apoptosis. J Biomed Sci 20: 100, 2013.

28. Nitulescu GM, Van De Venter M, Nitulescu G, Ungurianu A, Juzenas P, Peng Q, Olaru OT, Grădinaru D, Tsatsakis A, Tsoukalas D, et al: The Akt pathway in oncology therapy and beyond (review). Int J Oncol 53: 2319-2331, 2018.

29. Satake A, Takaoka M, Nishikawa M, Yuba M, Shibata $Y$, Okumura K, Kitano K, Tsutsui H, Fujii K, Kobuchi S, et al: Protective effect of 17beta-estradiol on ischemic acute renal failure through the PI3K/Akt/eNOS pathway. Kidney Int 73: 308-317, 2008.

30. Song G, Ouyang G and Bao S: The activation of Akt/PKB signaling pathway and cell survival. J Cell Mol Med 9: 59-71, 2005.

31. Zhang J, Liu XB, Cheng C, Xu DL, Lu QH and Ji XP: Rho-kinase inhibition is involved in the activation of PI3-kinase/Akt during ischemic-preconditioning-induced cardiomyocyte apoptosis. Int J Clin Exp Med 7: 4107-4114, 2014.

32. Zhang SB, Liu TJ, Pu GH, Li BY, Gao XZ and Han XL: MicroRNA-374 exerts protective effects by inhibiting SP1 through activating the PI3K/Akt pathway in rat models of myocardial ischemia-reperfusion after sevoflurane preconditioning. Cell Physiol Biochem 46: 1455-1470, 2018. 
33. Liu X, Hong Q, Wang Z, Yu Y, Zou X and Xu L: miR-21 inhibits autophagy by targeting Rab1la in renal ischemia/reperfusion. Exp Cell Res 338: 64-69, 2015.

34. Zhou M, Cai J, Tang Y and Zhao Q: miR-17-92 cluster is a novel regulatory gene of cardiac ischemic/reperfusion injury. Med Hypotheses 81: 108-110, 2013.

35. Hu Q, Luo W, Huang L, Huang R and Chen R: Apoptosis-related microRNA changes in the right atrium induced by remote ischemic perconditioning during valve replacement surgery. Sci Rep 6: 18959, 2016.

36. Chen J, Huang ZP, Seok HY, Ding J, Kataoka M, Zhang Z, Hu X, Wang G, Lin Z, Wang S, et al: mir-17-92 cluster is required for and sufficient to induce cardiomyocyte proliferation in postnatal and adult hearts. Circ Res 112: 1557-1566, 2013.

37. Benhamou D, Labi V, Novak R, Dai I, Shafir-Alon S, Weiss A, Gaujoux R, Arnold R, Shen-Orr SS, Rajewsky K and Melamed D: A c-Myc/miR17-92/Pten axis controls PI3K-mediated positive and negative selection in B cell development and reconstitutes CD19 deficiency. Cell Rep 16: 419-431, 2016.

38. Ma Q, Peng Z, Wang L, Li Y, Wang K, Zheng J, Liang Z and Liu T: miR-19a correlates with poor prognosis of clear cell renal cell carcinoma patients via promoting cell proliferation and suppressing PTEN/SMAD4 expression. Int J Oncol 49: 2589-2599, 2016.
39. Liang Z, Pan Q, Zhang Z, Huang C, Yan Z, Zhang Y and Li J: MicroRNA-125a-5p controls the proliferation, apoptosis, migration and PTEN/MEK1/2/ERK1/2 signaling pathway in MCF-7 breast cancer cells. Mol Med Rep 20: 4507-4514, 2019.

40. Zheng W, Matei N, Pang J, Luo X, Song Z, Tang J and Zhang JH: Delayed recanalization at 3 days after permanent MCAO attenuates neuronal apoptosis through FGF21/FGFR1/PI3K/Caspase-3 pathway in rats. Exp Neurol 320: 113007, 2019.

41. Yamamura S, Saini S, Majid S, Hirata H, Ueno K, Chang I, Tanaka Y, Gupta A and Dahiya R: MicroRNA-34a suppresses malignant transformation by targeting c-Myc transcriptional complexes in human renal cell carcinoma. Carcinogenesis 33: 294-300, 2012.

This work is licensed under a Creative Commons Attribution-NonCommercial-NoDerivatives 4.0 International (CC BY-NC-ND 4.0) License. 